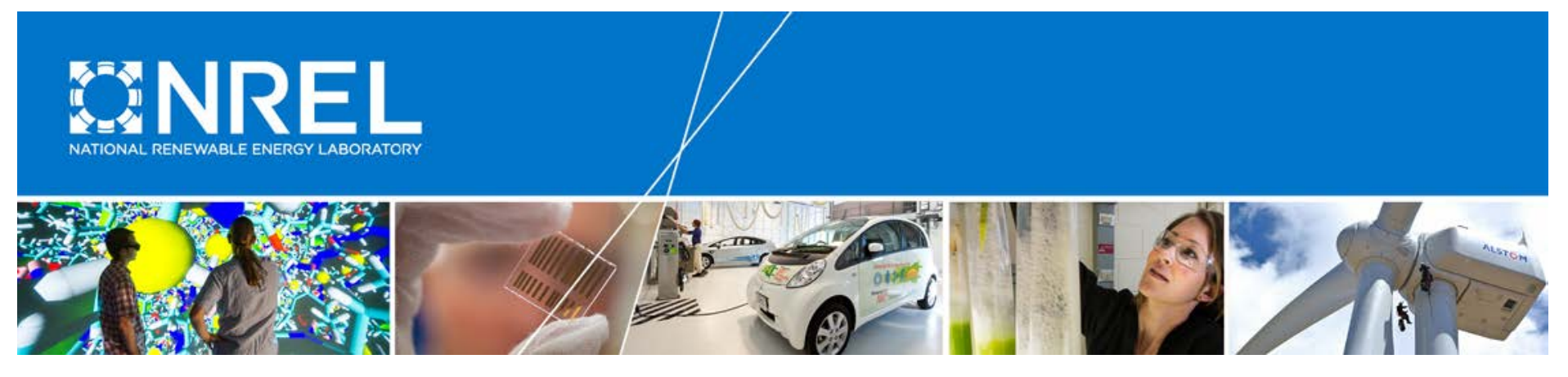

\title{
Developing Use Cases for Evaluation of ADMS Applications to Accelerate Technology Adoption
}

\section{Preprint}

Santosh Veda, Hongyu Wu, Maurice Martin, and Murali Baggu National Renewable Energy Laboratory

Presented at the IEEE Green Technologies Conference Denver, Colorado

March 29-31, 2017

(C) 2017 IEEE. Personal use of this material is permitted. Permission from IEEE must be obtained for all other uses, in any current or future media, including reprinting/republishing this material for advertising or promotional purposes, creating new collective works, for resale or redistribution to servers or lists, or reuse of any copyrighted component of this work in other works.

NREL is a national laboratory of the U.S. Department of Energy Office of Energy Efficiency \& Renewable Energy Operated by the Alliance for Sustainable Energy, LLC

This report is available at no cost from the National Renewable Energy Laboratory (NREL) at www.nrel.gov/publications.

\section{Conference Paper}

NREL/CP-5D00-67614

May 2017 


\section{NOTICE}

The submitted manuscript has been offered by an employee of the Alliance for Sustainable Energy, LLC (Alliance), a contractor of the US Government under Contract No. DE-AC36-08GO28308. Accordingly, the US Government and Alliance retain a nonexclusive royalty-free license to publish or reproduce the published form of this contribution, or allow others to do so, for US Government purposes.

This report was prepared as an account of work sponsored by an agency of the United States government. Neither the United States government nor any agency thereof, nor any of their employees, makes any warranty, express or implied, or assumes any legal liability or responsibility for the accuracy, completeness, or usefulness of any information, apparatus, product, or process disclosed, or represents that its use would not infringe privately owned rights. Reference herein to any specific commercial product, process, or service by trade name, trademark, manufacturer, or otherwise does not necessarily constitute or imply its endorsement, recommendation, or favoring by the United States government or any agency thereof. The views and opinions of authors expressed herein do not necessarily state or reflect those of the United States government or any agency thereof.

This report is available at no cost from the National Renewable Energy Laboratory (NREL) at www.nrel.gov/publications.

Available electronically at SciTech Connect http:/www.osti.gov/scitech

Available for a processing fee to U.S. Department of Energy and its contractors, in paper, from:

U.S. Department of Energy

Office of Scientific and Technical Information

P.O. Box 62

Oak Ridge, TN 37831-0062

OSTI http://www.osti.gov

Phone: 865.576.8401

Fax: 865.576.5728

Email: reports@osti.gov

Available for sale to the public, in paper, from:

U.S. Department of Commerce

National Technical Information Service

5301 Shawnee Road

Alexandria, VA 22312

NTIS http://www.ntis.gov

Phone: 800.553 .6847 or 703.605 .6000

Fax: 703.605.6900

Email: orders@ntis.gov 


\title{
Developing Use Cases for the Evaluation of ADMS Applications to Accelerate Technology Adoption
}

\author{
Santosh Veda, IEEE Member, Hongyu Wu, IEEE Senior Member, Maurice Martin, and Murali Baggu, \\ IEEE Senior Member
}

\begin{abstract}
Grid modernization for distribution systems comprises the ability to effectively monitor and manage unplanned events while ensuring reliable operation. The integration of distributed energy resources and the proliferation of autonomous smart controllers such as microgrids and smart inverters in distribution networks challenges the status quo of distribution system operation. Advanced distribution management system (ADMS) technologies are being increasingly deployed to manage the complexities of operating distribution systems. The ability to evaluate the ADMS applications in specific utility environments and for future scenarios will accelerate wider adoption of the ADMS and will lower the risks and costs of their implementation. This paper addresses the first step: to identify and define the use cases for evaluating these applications. The applications that are selected for this discussion include voltVAr optimization (VVO), fault location isolation and service restoration (FLISR), online power flow (OLPF) and distribution system state estimation (DSSE), and market participation. A technical description and general operational requirements for each of these applications is presented. The test scenarios that are most relevant to the utility challenges are also addressed.
\end{abstract}

Keywords-ADMS, VVO, FLISR, DSSE, OLPF, DSO, markets, distribution, DER, use cases, testing, test bed.

\section{INTRODUCTION}

Distribution networks are becoming as complex as the transmission part of the electric power grid. Some of these complexities arise from factors such as increasing penetration levels of renewable generation, the proliferation of autonomous control systems such as microgrids, the presence of grid-forming smart assets such as battery energy storage systems (BESS) and smart inverters, proposed market and regulatory changes that enable distribution markets and market aggregators, and the paradigm shift in grid operations wherein demand is being increasingly considered as a controllable asset. In addition to being critical to grid modernization efforts [1], advanced distribution management systems (ADMS) have become the primary decision support system for utility operators to handle these increasing complexities. The high capital costs and long lead times associated with installing an

S. Veda, M. Martin, and M. Baggu are with the Power Systems Engineering Center of NREL, Golden, Colorado, 80401, USA (e-mail: Santosh.Veda@nrel.gov).

$\mathrm{H}$. Wu is with Department of Electrical and Computer Engineering at Kansas State University, Manhattan, Kansas, 66506.
ADMS, even on a pilot basis, and the fact that its actual implementation needs to be tailored to the specifics of each individual utility make the adoption of ADMS a strategic venture. [2] Herein comes the critical need for evaluating the different ADMS applications for a specific utility network. Such efforts not only enable the characterization of performance and the quantification of costs/benefits but also provide insight into considerations such as instrumentation and data requirements for successful implementation. An evaluation will provide valuable insight for choosing the correct application for the intended operational objectives.

Another aspect that requires attention is the presence of autonomous controllers and their interaction with system operations. A demand response automation server (DRAS) acts as an aggregator with direct control of loads inside the premises of participating customers. Distributed energy resource management systems (DERMS) enable functions such as forecasting and control of output for a fleet of distributed energy resources (DERs). Microgrid controllers are capable of managing their local loads and generation assets and disconnect or reconnect to the bulk grid in the event of grid disturbances; thus, microgrid controllers can greatly alter the loading on the feeder and hence the operational performance of ADMS applications. Grid edge controllers [3] are also becoming commonplace; they control devices at the very end of the feeders at low-voltage levels to achieve control objectives such as voltage control and power quality.

Although a number of studies-e.g., [4], [5], [6], [7]-have discussed various ADMS applications, this paper presents these applications from the perspective of use cases for their evaluation. This approach allows for raising pertinent questions that are of interest to utility operators as they explore the adoption of these technologies. For each of the selected ADMS applications, specific use cases that are of interest to the utilities are addressed. For example, a use case could be an evaluation of the performance of a selected ADMS application in the presence of high penetration levels of renewables. This use case could be further modified to include various penetration levels of DERs, a mix of DERs, the capabilities of the DERs, etc. These scenarios can be tailored to the needs of a specific stakeholder. For example, a vendor could use these use cases to calibrate the settings for different applications or demonstrate benefits, whereas a utility could use these use cases to evaluate implementation readiness and future grid scenarios. 
The ADMS applications that are addressed in this paper include volt-VAr optimization (VVO); fault location, isolation, and service restoration (FLISR); online power flow (OLPF) and distribution system state estimation (DSSE); and market participation. Of several ADMS applications [8], these four were selected to represent two applications that are critical to current utility operations: FLISR and VVO; one core ADMS functionality (OLPF/DSSE); and one as a future need: market participation.

This paper is organized as follows. Section II provides a technical description, general requirements, and use cases for the VVO application. Similarly, Section III, Section IV, and Section V provide descriptions and use cases for the FLISR, OLPF/DSSE, and market participation applications, respectively. Section VI concludes.

\section{VOLT-VAR OPTIMIZATION}

The primary objective of the VVO application is to maintain acceptable voltage levels throughout the feeder in addition to other objectives such as power factor correction, loss minimization, energy efficiency, and peak demand shaving. The VVO function achieves these objectives by using necessary grid measurements and then optimizing the set points of voltage control devices such as capacitor banks and voltage regulators. In addition to these traditional utility devices, other controllers such as smart inverters, microgrid controllers, DRAS/DERMS, and grid-edge devices may be leveraged by the VVO application for optimal performance. The VVO function should minimize the voltage regulator and capacitor bank operations to limit the wear and tear on these utility assets, especially in the event of voltage fluctuations caused by intermittency from high penetrations of solar photovoltaic (PV).

\section{A. General Requirements}

The VVO function in traditional distribution feeders needs to observe only slow dynamics, but this is not the case in a distribution network with a high penetration level of DERs. Given sub-second dynamics due to feeder switching and flow control, fluctuations caused by intermittent generation sources such as wind and solar, and interactions with responsive loads, the process of regulating and stabilizing voltage and reactive power will need to operate at faster timescales. Although the VVO itself does not need to operate at the timescales of these events, it should be able to manage these fast-response assets through hierarchical or distributed controls. To implement the VVO, the ADMS should obtain real-time voltage measurements from multiple sensors, which may include advanced metering infrastructure (AMI). The ADMS should have sufficient observability of the distribution network, especially because the voltage profile for feeders with renewables cannot be estimated with only a few measurements. It should be guaranteed that no control action that would produce unacceptable voltage or loading conditions should be requested by the ADMS under any circumstance. The VVO should verify that its control actions are indeed implemented by the field devices, and it should initiate remedial control actions if the original actions are not implemented. The VVO implementation should fail gracefully in the event of a fatal incident, such as the loss of communication or inaccurate measurements, allowing the field devices to revert to local control. If the VVO scheme uses a distribution network model, it should ensure that the model is updated when there is any change in feeder configuration. The VVO scheme should also be able to minimize the impact of DER variability on grid assets. Further, the VVO scheme should be capable of gauging the impact of assets such as energy storage and electric vehicle loads on the voltage profile in addition to utilizing forecasts for intermittent DERs. The VVO functionality should also be able to interact with other autonomous control systems that are capable of regulating voltage, such as microgrids, smart inverters, DERMS, and grid-edge devices.

\section{B. Use Cases}

The following cases are used to evaluate the VVO application.

\section{1) Voltage Regulation}

The primary objective of any voltage control scheme is to maintain an acceptable voltage profile throughout the distribution network without violating the voltage at any node in the network. Although the range of acceptable voltage magnitudes as defined by the American National Standards Institute (ANSI) is between $95 \%$ and $105 \%$ of the rated voltage, the utility customer may require a narrower range with the objective of voltage flattening. The VVO scheme should be capable of meeting this requirement. The presence of DERs downstream and their intermittency will impact the ability of any VVO to achieve this objective due to real power and/or reactive power injection. The performance should be characterized in the presence of different types and penetration levels of assets such as a) legacy voltage control equipment, b) smart inverters, c) storage assets, d) grid-edge devices, and e) autonomous local controls such as DERMS/microgrids.

\section{2) Peak Load Management}

Another important objective for the VVO function is peak load reduction. In volt-VAr parlance, load reduction is achieved through a technique called the "conservation voltage reduction" (CVR). [9] In CVR, the feeder voltage magnitude is reduced to the lowest allowable value without incurring voltage violations. This reduces the power consumed by voltage-dependent static loads that are characterized as constant impedance and constant current loads. Although the power consumed by constant power loads (such as electronic loads) remains the same, the current drawn will increase as the voltage reduces. The ability of the VVO to provide significant CVR benefits is limited by the nature of the feeder itself. For example, any voltage reduction on a hypothetical feeder with $100 \%$ constant power loads will lead to an increased current draw and the associated line losses, thereby defeating the objective of load reduction.

The VVO function can be used to reduce peak load on a feeder for energy-efficiency and investment-deferral objectives, making it an attractive option for managing heavily loaded feeders. A use case of interest may be to study the performance of the VVO scheme accounting for the feeder characteristics. The ability of the VVO to coordinate with "aggregator-type" controls (such as DRAS and DERMS) and 
downstream controls (such as microgrid and grid-edge controllers) will be critical to achieve these objectives.

\section{3) Performance Characterization}

As with many advanced grid functions, it can be difficult to directly evaluate the performance of a VVO system because the objectives are fluid. The VVO may be working to minimize some weighted sum of objectives, including energy loss, MW load, voltage violation, or current violation; therefore, an important use case for the VVO application is to characterize its performance. Performance must be measured by:

- Examining the instructions to the VVO (the weighted objectives)

- Using those instructions to determine what the VVO should have done in terms of meeting those objectives (ideal outcome)

- Compare both the ideal outcome and the actual outcome to a baseline representing what would have happened in the absence of the VVO.

It is almost certain that a VVO system would do better with some sets of weighted objectives than others; therefore, the performance evaluation must be done multiple times with different sets of weighted objectives, with enough variation in the weighted objective combinations to obtain a reasonable sense of how the system will perform in an operational setting.

These types of iterations (wherein a baseline, ideal, and actual outcome must be produced) are ideally suited for a testbed environment. Changes to the VVO algorithm can also be explored this way.

In addition to technical performance, the benefits from implementing a VVO scheme should be studied. Some of the benefits include deferred capital expenditures and improvement in asset utilization, reduced environmental impacts, improved efficiency and flexibility in utility operations, and improved reliability and resiliency [10]. These benefits will vary depending on the specific utility network and VVO configuration that is implemented. Quantifying and characterizing benefits enables the selection of the optimum VVO configuration for the selected distribution network before actual field deployment.

\section{4) Integration of Active Grid-Edge Devices}

The complexity of VVO operations increases if there are other autonomous voltage-regulating schemes. An example might be a scheme that uses grid-edge devices for voltage regulation at the lowest voltage levels, which provide dynamic VAR control based on local conditions and without communication.

In the best-case scenarios, the reactive devices would help the system balance itself; however, it is easy to imagine a scenario wherein the VVO and the autonomous reactive devices are working against each other. When the system operator selects a new set of weighted objectives, the behavior of the VVO would change, but the behavior of the autonomous voltage control schemes would not. The activities of the two might reinforce each other, or they may conflict in unexpected ways.

Evaluation efforts could answer a number of questions related to this use case. Under what circumstances will the $\mathrm{VVO}$ and the autonomous reactive devices work against each other? Can the VVO algorithm be tuned to work better with autonomous reactive devices? Can the placement of sensors in intermediate points along the feeder provide information that will allow the VVO to work better with the devices? What is the optimal way to allocate regulation responsibilities between the VVO and other such voltage control schemes?

\section{5) Evaluation of Different Control Architectures}

Control architectures present a number of interesting challenges with regard to VVO. First, consider whether the VVO is controlled centrally or in a distributed fashion. Consider also the situation in which a DER, responding to local measurements, needs one voltage, whereas at the utility level the VVO decides that another voltage is needed. Or, what if the DER and the VVO both decide to lower the voltage at the same time? Ultimately, control must be coordinated at several levels: transmission, sub-transmission, distribution, and microgrid/DER. The evaluation should provide opportunities to try combinations of the control algorithms at each of these levels and for different control architectures.

\section{FAUlt Location, Isolation, AND SERVICE RESTORATION}

FLISR is one of the major applications associated with distribution system modernization. It is used to mitigate the impact of faults in a distribution system, especially permanent faults (faults that are not self-clearing), which induce enduring influence on the other parts of the system.

The first objective of the FLISR application is to detect permanent faults on the distribution feeder and determine the approximate location of the fault, i.e., the faulted section of the feeder that is bounded by two or more feeder switches. Meanwhile, it should isolate the faulted section of the feeder and close normally open tie switches to transfer loads that are downstream of the faulted section. In addition, FLISR is designed to prevent service restoration when alternate circuits do not have enough capacity to carry additional load because this could lead to overloading those circuits, which could lead to further deterioration in operation.

The benefits of FLISR include reducing the outage duration and number of customers interrupted, i.e., by improving the system average interruption duration index (SAIDI) and the system average interruption frequency index (SAIFI). The improvements are because some customers will be restored to service in less time than the threshold for permanent outages (usually 5 minutes). FLISR schemes implemented as part of the Smart Grid Investment Grant program have shown a reduction of $55 \%$ in the number of customers interrupted and $50 \%$ in the customer minutes of interruption for partial feeder outages.[11]

\section{A. General Requirements}

In general, the ADMS and the outage management system (OMS) should analyze all available real-time information acquired from field devices to detect faults and other circuit conditions for which service restoration actions are required. Information typically used includes the outputs of fault detectors - e.g., relays, fault magnitude at various locations on the feeder, feeder segment and customer meter energization status, and protective relay targets. 
All control actions identified by the centralized FLISR may be automatically executed by issuing supervisory control commands to substation circuit breakers and various feeder switching devices-e.g., reclosers, load breaker switches, and sectionalizers - that are equipped with supervisory control capabilities. Note that the FLISR function should operate only for permanent faults occurring on the portion of the feeder that causes the sustained loss of electrical service on the circuit; temporary faults that are cleared without a sustained loss of service by standard automatic reclosing schemes should not result in the execution of FLISR control actions.

The FLISR function should not attempt to perform control actions to restore service loss resulting from blown fuses on feeder laterals, emergency load-shedding activities, and manual feeder tripping; however, it should be aware of these situations when picking up loads. Before executing any downstream service restoration actions, the ADMS should confirm that the alternative source is energized and able to accommodate the additional load being switched. Service restoration actions performed or recommended by the distribution management system should not produce undesirable electrical conditions, such as current overloading of conductor and equipment, high/low customer voltage situations, and closing into a permanent fault.

\section{B. Use Cases}

\section{1) High Penetrations of DERs}

The traditional distribution system is a passive system in a radial configuration with unidirectional power flow from the substation to the customer. With no DERs integrated, implementing the electrical distribution protection scheme and the operation of FLISR are relatively easier compared to cases with DER penetration. Also, as the penetration level of DERs increases, the complexity of the protection scheme and operation of FLISR increases. Specifically, the isolation and restoration functionality must take into account the energy sources that are located downstream within the distribution system. These DERs could be either at a commercial and industrial customer side or distributed, feeding the fault from the other end of the distribution system. The line loading before and after a permanent outage may be drastically different because of the tripping of the DERs from the loss of the grid.

Contributing to the challenges of this use case are intermittency and visibility. The intermittency of power available at the DER interconnection points makes the selection of alternate feeders more difficult because the FLISR does not know how much power will be required or how much is available. To solve this problem, the FLISR function should use modeling to estimate the available output power from the DERs on alternative feeders. The FLISR function should also take into account the uncertainly factor caused by forecasted errors.

The FLISR also needs visibility into the amount of power being produced by the DER at any given moment. This implies some kind of communication channel between the DER and the FLISR system. It may also be necessary for the FLISR to be able to send on/off commands to the DER so that the power is not being added to the feeder in a case when doing so would be problematic, such as if the anti-islanding conditions have not already disconnected the DER. Finally, the FLISR would need to have a relatively fast response time to accommodate situations where the status of the fault(s) and output from the DERs are changing rapidly.

Because DERs are active devices that can inject power into the distribution system and trigger undesired protective behaviors or, worse, create abnormal situations during restoration, the most dangerous condition is when a DER source has not been islanded from the distribution system and will back-feed through the secondary transformer, resulting in a primary voltage rise on a conductor thought to be deenergized. It is necessary to coordinate the settings of the relays, sectionalizers, and other protective devices to avoid this type of false tripping or unintended exposure of service personnel to energized conductors.

In the event of unintentional islanding, the anti-islanding provisions of IEEE 1547 apply. Anti-islanding trigger points should be built into the DER or microgrid controller while accounting for ride-through capabilities that are envisioned for future DERs.

\section{2) Interaction with Microgrids}

The interaction of FLISR with microgrids needs to be understood due to the microgrid controller's greater autonomy to control its load and generation in addition to its ability to ride-through faults and reconnect to the distribution grid when it stabilizes. Protection coordination and discerning actual load from net load are of interest to ensure smooth transitions between outage and normal states for both the microgrid and the distribution grid.

If the DERs are managed by a microgrid, the impact of the DERs becomes much easier for FLISR to manage. The microgrid controller can control the devices inside the microgrid to achieve power balance so that the intermittency can be managed locally. It is also responsible for maintaining the desired power quality and availability at the point of interconnection (POI). This is true for both islanded and gridconnected operation modes.

However, when the penetration level of intermittent DERs increases, microgrids may require effective isolation mechanisms to transition the microgrid to an islanded mode during a fault condition that is either internal or external to it to minimize its impact on the external grid. This creates additional complexity of integrating and coordinating the microgrid controller with the DMS and OMS.

\section{3) Very High Loading Conditions}

In situations when the feeder load exceeds $50 \%$ capacity, the effectiveness of the FLISR schemes is limited, and it may even be detrimental to the objective of minimizing service interruption. The selection of alternate feeders requires greater care because as any errors in redirecting power flow are more likely to have damaging consequences for the system.

In the case of cold-load pickup, FLISR will need to be able to model the power flow and estimate the additional demand and the duration of that demand. Because protective relays can interpret cold-load conditions as faults, the FLISR will need to change protective relay settings for the duration of the coldload pickup to avoid de-energization of the healthy feeder. 


\section{Online Power Flow/Distribution System State ESTIMATION}

OLPF and DSSE are core applications that provide situational awareness for the operator and for other applications, such as VVO and FLISR. Although the objective of OLPF and DSSE are the same - to obtain system states (voltage phasors) at every point in the network-they are achieved differently. Both applications use a model of the distribution network to relate the measurements reported by sensors located across the network. OLPF relies on the physics-based electrical model to compute the voltages at all the nodes; DSSE takes into account the availability of sufficient measurements to observe the system states and the uncertainties around measurement accuracies. DSSE is a datafitting tool that ensures that the ADMS has as accurate estimate of the system states given non-ideal measurement systems and data quality

\section{A. General Requirements}

In general, OLPF requires a model of the distribution system as well as feeder measurements of both real and reactive power at strategic points in the system. The simplest scenario is for the supervisory control and data acquisition system to provide this information. It also needs to know the status of all control devices on the distribution system. The OLPF should be capable of discerning any changes in topology and computing the system states whenever necessary. With the proliferation of newer measurement sensors such as smart meters and micro-phasor measurement units ( $\mu$ PMUs), the DSSE should be able to take these measurements along with traditional sensors. The DSSE should be capable of integrating a wide variety of sensors with different reporting rates and managing varying data quality when estimating the system states. The DSSE should also be capable of recognizing loss of observability due to bad data or loss of communication and adjust its estimation methodology.

\section{B. Use Cases}

\section{1) Calibrate the OLPF/DSSE Functions}

The ADMS evaluation will afford an opportunity to precisely calibrate the OLPF/DSSE functionality. The output of these functions can be compared to actual measurements from the evaluation tests to determine variance. The algorithms can then be tuned in an iterative fashion to obtain the best match. This distribution system can then be changed, and the interactive process can be run again to further tune the algorithm and verify that it performs well on a variety of distribution configurations. Another use case could be to characterize the performance of these algorithms in the presence of high-noise measurements, lack of complete system observability, and addition of multiple types of sensors. Because they are critical applications, these functions can also be evaluated on their "graceful degradation" of performance in the event of component failures.

2) Measure Performance with a Hierarchy of Distributed Sensing

An important use case is to understand the impact of modern sensors and smart assets on enhancing the performance of OLPF/DSSE algorithms. Several efforts at multiple levels have been undertaken on integrating evolving technologies into mainstream ADMS applications. Some of these evolving technologies include OpenFMB [12]; OpenADR [13]; and smart grid-edge devices such as smart inverters, microgrid controllers, and high-resolution, highfidelity sensors such as distribution PMUs ( $\mu$ PMUs). Although these technologies are promising, there is a need to characterize the performance and quantify the benefits of enhancing the ADMS applications with these technologies. This would enable the utilities to adopt such technologies with greater confidence while providing valuable insights to application/technology developers on practical considerations such as scalability and data quality challenges.

3) Minimize Customer Impact from Planned Outages

OLPF/DSSE, as core applications, are critical to achieving the objectives of other advanced functions, such as FLISR and switching order management for servicing power system components for outages and maintenance, respectively. Although contingency analysis is a critical tool for transmission system operations, a similar "what if" analysis can be used in the distribution system to determine the optimal switching order of power system components that need to be serviced for maintenance or after a failure. OLPF/DSSE can be used to calculate not only the status of the current distribution system but also its possible status based on changes. If an outage is necessary for an equipment repair or upgrade, these core applications can identify ways in which it can be done that will minimize the number of customers impacted. By accounting for the expected response of microgrids and DRAS, OLPF/DSSE can more accurately estimate the impact of a planned outage, thereby enabling the most optimum circuit configuration. When they are required to perform scheduled component outages, the distribution operators may be able to leverage the islanding and off-grid operational capability of a microgrid to minimize the number of customers affected and the duration of the interruption.

4) Modeling Loss of PV

OLPF/DSSE can be used to provide insight into behind-themeter load components. Such a capability will be critical in distribution feeders that have a high penetration level of PV and thus there is a need for the operator to be aware of the actual load and the PV output instead of only the net feeder loading. This would be useful when trying to determine the impact from, for instance, a sudden loss in PV generation. Such a capability would require that the OLPF/DSSE functions leverage other functions such as load and PV forecasting, data on customer facility installations, load models, and AMI meter data.

\section{MARKET PARTICIPATION}

Market participation includes a broad set of applications that involve the interaction of the distribution system with energy markets. The applications consist of participation in bulk services that currently have a market available already or future services that involve a still-evolving market design based on a distribution service operator (DSO). In each of these markets, the distribution network (or some part of it) is expected to execute some well-defined response. From the ADMS perspective, these market functions will enable the 
distribution system operator to participate in a market (such as a market participant in the current framework of an independent system operator [ISO] market) or provide a platform for other aggregators and customers to participate in a market (the equivalent of an ISO for distribution network). An example of such an application is the demand response program, wherein the grid operator can curtail/schedule customer loads in exchange for monetary incentives.

\section{A. General Requirements}

Depending on the market structure, market participation application in an ADMS could have different sets of requirements. At a basic level, the market function should be able to communicate set points or control parameters to the assets that are participating in the market. The function should also be able to ascertain the response of the individual assets, thereby ensuring that the output was compliant with the expected response. The market function should also be able to correct for any aberration between expected and actual responses through additional control actions. The market function should be able to determine the monetary compensation for all the players based on the construct of the market; this is a critical functionality, especially when the value provided is determined based on performance. With the proliferation of smart controllers such as smart inverters, microgrids, and DERMS/DRAS, the market functions would be required to interact with these controllers as well. In addition to these functional requirements, the market function may be required to archive market transaction information efficiently.

\section{B. Use Cases}

\section{1) Maintaining Power Quality While Providing Services to} the Bulk Grid

A possible market function could be the distribution operator coordinating the provision of bulk services while maintaining quality of service. One example is when the DMS operator is required to reduce system loading. A less favorable option would be to curtail loads, which leads to the deterioration in the quality of service or outright disruption. Alternate means would be deploying assets such as DERs, storage, or demand response to achieve system demand reduction. An important factor in such a scenario is accounting for the capabilities of different assets, especially when transitioning from local control to coordinated central control. Such transitions should not adversely affect the power quality in the local area where the asset is located.

\section{2) Distribution System Operators Providing Market Functions}

DSOs are grid entities that are envisaged to carry out functions similar to those of an ISO at the bulk transmission level. A key function for a DSO would be to create a market framework that enables the participation of stakeholders such as local utilities, aggregators, DER owners, end-use customers, and local utilities. The interaction of the DSO with the assets would be through pricing signals. The DSO should also be able to quantify the performance of all the assets and ensure that the performance objectives are met through corrective actions when the initial response is not satisfactory.

\section{3) Estimating Available Capacity for Bidding in Energy} Markets

When the distribution operator wishes to participate in the bulk market, it is necessary that the ADMS market functions are capable of estimating the available capacity across the distribution network. The operator may then bid into the reserve market based on the estimated available capacity. Use cases may include scenarios where the role of the market aggregators in market efficiency will be studied. The performance of the market functions for different loading levels, different load types, and different bulk price levels can also be characterized.

\section{CONCLUSION}

ADMS technologies offer tremendous latitude for grid modernization for distribution systems. The reliability improvements and cost-effective operations offered by the ADMS technologies are becoming increasingly indispensable to the operation of distribution networks that have increasing system complexity. These ADMS technologies can usher in new technology, policy and market frameworks that are required for managing future distribution networks. Given the high capital costs and technological risks associated with implementing an ADMS, there is a critical need for evaluating these applications for the early identification of adoption challenges, technical risks, cost and benefits characterization, and effectiveness of the selected application for a specific utility environment. This paper identifies and addresses the first step toward developing the ability to evaluate these systems. Further work is needed to develop test objectives for each of these scenarios and develop comprehensive test plans. The approach should be tailored to a specific utility environment and a specific vendor's ADMS. The lessons from this effort can be used to further tune the use cases and scenarios.

\section{ACKNOWLEDGEMENTS}

This work was supported by the U.S. Department of Energy under Contract No. DE-AC36-08GO28308 with the National Renewable Energy Laboratory. Funding provided by the U.S. DOE Office of Electricity Delivery and Energy Reliability, as part of the Grid Modernization Laboratory Consortium. The U.S. Government retains and the publisher, by accepting the article for publication, acknowledges that the U.S. Government retains a nonexclusive, paid-up, irrevocable, worldwide license to publish or reproduce the published form of this work, or allow others to do so, for U.S. Government purposes.

The authors acknowledge the project team members for valuable discussions. In addition to those from the National Renewable Energy Laboratory (NREL), the project team consists of participants from the following organizations: Argonne National Laboratory, Pacific Northwest National Laboratory, the Electric Power Research Institute, Schneider Electric, General Electric, and OPAL-RT. 


\section{REFERENCES}

[1] Jianhui Wang, "ADMS for Grid Modernization Importance of DMS for Distribution Grid Modernization," Argonne National Laboratory, ANL/ESD-15/16, Sep. 2015.

[2] National Renewable Energy Laboratory (NREL), "Insights into Advanced Distribution Management Systems," US Department of Energy, Feb. 2015.

[3] "Varentec Grid Edge Management Systems," Varentec. [Online]. Available: http://varentec.com/products/gems/. [Accessed: 16-Dec-2016].

[4] Jianhui Wang, Xiaonan Lu, James Reilly, and Sal Martino, "ADMS for Grid Modernization - High-Level Use Cases for DMS," Argonne National Laboratory, ANL/ESD-15/18, Sep. 2015.

[5] Electric Power Research Institute (EPRI), "Smart Distribution Applications for Distributed Energy Resources: Distribution Management System Use Cases," 22-Dec-2013. [Online]. Available: http://www.epri.com/abstracts/Pages/ProductAbstract.asp $\mathrm{x}$ ?ProductId $=000000003002002464$.

[6] Frances Cleveland, "CEC Value of DA: Distrbution Automation Detailed Scenarios," May-2007.

[7] "Use Cases for Smart Grid," International Telecommunication Union Work Group, Geneva, Dec. 2011.

[8] Jianhui Wang, "ADMS for Grid Modernization - DMS Functions," Argonne National Laboratory, ANL/ESD15/17, Sep. 2015.

[9] Bob Uluski, "Volt/VAr Control and Optimization Concepts and Issues," 2011. [Online]. Available: http://cialab.ee.washington.edu/nwess/2012/talks/uluski.p df.

[10] Department of Energy (DOE), “Application of Automated Controls for Voltage and Reactive Power Management," Dec-2012. [Online]. Available: https://www.smartgrid.gov/files/VVO_Report_Final.pdf. [Accessed: 16-Dec-2016].

[11] ÜS Department of Energy, "FLISR Technologies Reduce Outage Impact and Duration," Dec-2014. [Online]. Available: https://www.smartgrid.gov/files/B5_draft_report-12-182014.pdf.

[12] SGIP, "Open Field Message Bus (OpenFMB)." [Online]. Available: http://www.sgip.org/openfmb/.

[13] OpenADR Alliance, "Open Automated Demand Response (OpenADR)." [Online]. Available: http://www.openadr.org/overview. 